\title{
A Post-coder Feedback Approach to Overcome Training Asymmetry in MIMO-TDD
}

\author{
Arun Kumar Miryala, Irina Merin Baby, Kumar Appaiah, Sibi Raj B. Pillai \\ Department of Electrical Engineering \\ Indian Institute of Technology Bombay, Mumbai, India
}

\begin{abstract}
Time Divison Duplex (TDD) wireless communication systems are inherently bidirectional, which facilitates exploiting channel reciprocity for pilot based channel estimation of both uplink and downlink. However, there exists a gross asymmetry in channel estimation complexity for the uplink and downlink, particularly for Multiple Input Multiple Output (MIMO) TDD systems. Usually, Base Stations (BS) with more antennas need to estimate fewer parameters from each antenna, whereas the estimation requirement is disproportionately higher at the User Equipment (UE). Unlike the UE, the BS has powerful hardware, computational resources and energy to accurately estimate and track channel profiles. To overcome this asymmetry, we propose a solution for MIMO-TDD downlink communication, wherein the post-coder part of the channel matrix is quantized at the BS, and is communicated to the UE via a low-rate channel. Using asymptotically tight lower bounds on the downlink achievable rates, we quantify the performance of the proposed scheme. Simulations reveal that a moderate number of quantization bits are sufficient to achieve rates close to the the link capacity. We further show that, when the BS has many more antennas at than the UE, the channel can be compensated by appropriate transmit domain precoding without post-coder knowledge at the UE.
\end{abstract}

\section{INTRODUCTION}

The use of Multiple Input Multiple Output (MIMO) techniques has been pioneered as one of the key approaches to enhance data rates of wireless systems. When the Base Station (BS) possesses a large number of antennas, the use of time-division duplex (TDD) communication with uplink training can significantly enhance downlink data rates and link reliability [1]. This concept has been explored in great detail and has been shown to work effectively in practice. However, most considerations assume the receiving user equipment (UE) to possess a single antenna. In such systems, the base station can effectively precode to compensate for channel effects, so that 
the receiver detection is significantly simplified. In particular, once the channel vector is known at the BS, knowing its norm at the UE is sufficient to decode the downlink transmissions. In other words, UE need not perform full fledged channel estimation using downlink pilots, since this is a waste of effort. However, the situation drastically changes when there are multiple antennas at the UE, since estimating the channel norm at each UE antenna is now insufficient for rate maximization. On the other hand, training to estimate the full channel is not only wasteful, but also an onerous task for a battery powered, low form-factor UE. Notice that the computational and hardware resources available at the BS allows it to near accurately estimate and track the uplink channel, where only a few parameters per antenna need to be estimated. The reverse link, through reciprocal in TDD, requires estimating several coefficients per antenna, that too with less resources, leading to a 'curse of asymmetry' in pilot based training. Moreover, different UEs may be equipped with varying number of antenna and hardware, making a single-size-fits-all pilot training infeasible and inaccurate. In order to address this, we propose a solution based on the feedback of partial channel state information (CSI) measured at the base station to the UE, and demonstrate that this enhances the system throughput significantly, without compromising on simplicity. Our feedback approach is particularly suitable for practical $r \times t$ MIMO systems where the number $t$ of antennas at BS ranges from 4 to 12, whereas the UE may be equipped with 2 to 4 antennas.

Typical wireless systems employ pre-processing of the transmitted symbols, leading to reduced receiver complexity as well as higher data rates [2]. Acquiring receiver CSI places a huge burden on the UE in Frequency Division Duplex (FDD) links, particularly for MIMO systems. The alternative TDD systems have scope for efficient workarounds, once the BS has acquired the full CSI vector of the reciprocal channel. It is of crucial importance to then convey the channel singular values as well as the left unitary matrix (called post-coder) of the downlink, under Singular Value Decomposition (SVD), to the UE. The low rate control channels present in the standards [3] can be exploited for such CSI feedback. Notice that the feedback bit budget is critical for block fading channels, where frequent channel updates using a small number of bits are required, while adaptive feedback can reduce this load for smoother channel variations. In any case, it is essential to understand the loss of performance due to the quantization of CSI.

Let us focus on conveying the post-coder matrix to the UE. This requires efficient quantization of unitary matrices. Typical approaches to quantize unitary matrices involve the use of vector quantization on manifolds, such as the Grassmannian and Stiefel manifolds. These approaches primarily exploit the algebraic structure of the precoder to obtain quantization schemes (unit 
vectors [4], semi unitary matrices [5] and unitary matrices [6]). Though these methods have been shown effective in theory, they involve operations on high dimensional manifolds, often an overkill due to the complex processing requirements. On the other hand, it is known that unitary matrices can be efficiently decomposed into independent parameters using Givens rotations and Householder transformations [7]. The utility of this decomposition is epitomized by their adoption in several wireless standards [8]. Moreover, the parameters obtained in this approach are independent and can be quantized using scalar quantizers. Another advantage is that in slowly varying channels, the temporal evolution of these scalar parameters can be tracked using single-bit adaptive quantizers [9].

The main objective of the current paper is to propose efficient post-coder quantization schemes for TDD MIMO using Givens rotation, which not only fit the bit budget, but also significantly enhance the downlink achievable rates. In order to characterize the quantizer performance, we perform an information theoretic analysis, assuming the availability of channel singular values and quantized post-coder CSI at the UE. We will show that rate gap between our scheme and the capacity with full CSI vanishes exponentially in the number of feedback bits. Furthermore, numerical computations reveal that a small number of feedback bits suffices to achieve rates close to the ergodic capacity using full CSI. The approaches suggested here are also attractive due to the fact that several standards already use the Givens rotations to quantize precoder information [8], and the enhancements proposed here can be realistically adopted in practical systems.

For UEs having a few antennas, channel estimation and feedback requirements may behave differently as we add more and more antennas at the BS. More specifically, channel hardening will have a big impact in systems with hundreds of transmit antennas. However, to reap the benefits of such massive MIMO systems, without the receiver having access to the post-coder, nonconvenional techniques may be required. For example, conventional water-filling based transmit power allocation approaches appear to perform worse in the absence of full receiver CSI. For completeness, we also propose efficient precoding based communication schemes for massive MIMO systems without post-coder information at the receiver, and analyze the resulting rates.

The remaining part of this paper is organized as follows: Section $\Pi$ describes the system model. In Section III], we propose an efficient post-coder quantization scheme, and analytically characterize the achievable rate performance as a function of the number of feedback bits. Furthermore, we propose schemes for massive MIMO with very limited feedback of CSI from the BS to the UE. Section IV numerically compares the performance for various MIMO configurations, and 
shows that the suggested schemes are very efficient. Finally, Section $\mathrm{V}$ concludes the paper with some future directions.

\section{System MODEL}

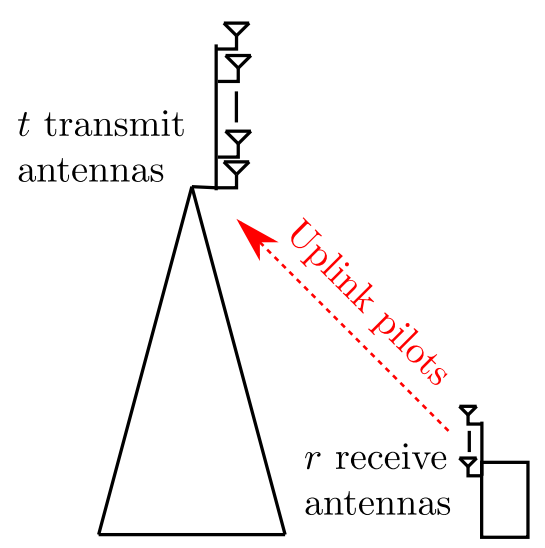

Step 1: Uplink training

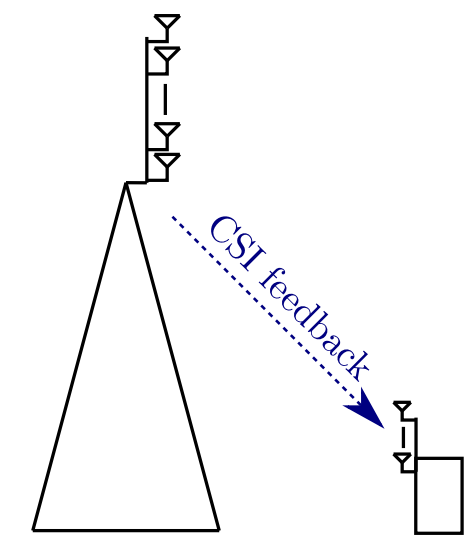

Step 2: Feed back quantized postcoder $(\hat{\mathbf{U}})$ to $\mathrm{UE}$

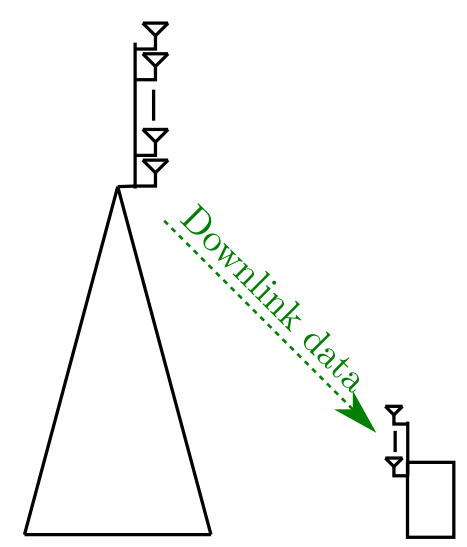

Step 3: Downlink transmission

Fig. 1: Training and feedback in the MIMO TDD system. The base station first learns the channel from uplink pilots (Step 1), and sends back just the compressed (as CSI feedback) post-coder to the UE (Step 2), which uses it to decode data (Step 3).

We consider a TDD-MIMO system with $t$ antennas at the transmitter and $r$ antennas at the receiver. In this system, the channel is estimated at the base station using uplink pilots, and due to channel reciprocity, the downlink channel is assumed to possess the same channel coefficients. We assume a block fading model with Rayleigh distributed channel coefficients, wherein the MIMO channel is modeled by a channel matrix $\mathbf{H} \in \mathbb{C}^{r \times t}$ whose entries are i.i.d. unit normal complex Gaussian random variables. That is, when the input $\tilde{\mathbf{x}} \in \mathbb{C}^{t}$ is sent by the transmitter (base-station) to the receiver (UE), the received symbols $\tilde{\mathbf{y}} \in \mathbb{C}^{r}$ are given by

$$
\tilde{\mathbf{y}}=\mathbf{H} \tilde{\mathbf{x}}+\eta
$$

Here $\eta \in \mathbb{C}^{r}$ is the additive white Gaussian noise vector with i.i.d. entries, each having unit variance. We assume that $t>r$, thus making the $\operatorname{rank}(\mathbf{H})=r$ with high probability. Due to reciprocity, the channel from the UE to the BS is then $\mathbf{H}^{T}[1]$. The singular value decomposition (SVD) of the $\mathbf{H}$ is given by $\mathbf{H}=\mathbf{U} \Sigma \mathbf{V}^{\dagger}$ where $\mathbf{U} \in \mathbb{C}^{r \times r}$ and $\mathbf{V} \in \mathbb{C}^{t \times r}$ are unitary matrices and $\Sigma \in \mathbb{R}^{r \times r}$ contains the non-negative singular values $\sigma_{1} \geq \cdots \geq \sigma_{r}$ of $\mathbf{H}$. Also, an average transmit power constraint is imposed by stipulating $\mathbb{E}\left[\tilde{\mathbf{x}}^{\dagger} \tilde{\mathbf{x}}\right] \leq P_{T}$, where the expectation is over the transmitted codewords, across fading blocks. 
As shown in Fig. 1, the transmission within each coherence interval occurs in three steps [1]. The first step involves uplink training for channel estimation. Here, the pilot symbols $\mathbf{p}_{1}, \mathbf{p}_{2}, \ldots \mathbf{p}_{\tau}$ (all belonging to $\mathbb{C}^{r}$ ), are sent over $\tau$ time instances. The signal received at the base station when the UE transmits these pilots is described by

$$
\mathbf{y}_{B, i}=\mathbf{H}^{T} \mathbf{p}_{i}+\eta_{B, i}, \quad i=1,2, \ldots \tau
$$

The channel can then be estimated at the base station using $\mathbf{y}_{B, i}, i=1, \cdots, \tau$. We assume that the CSI obtained by this approach is error-free, i.e. full CSI at Transmitter (CSIT). If full CSI is also available at Reciever (CSIR), then the capacity of this model is well known [10-12]. In particular, $r$ parallel channels are effectively available in each fading block [10], and the average transmit power is optimally divided over these parallel channels, and also across fading blocks, to achieve the ergodic capacity. Observe that once the BS knows $\mathbf{H}=\mathbf{U} \Sigma \mathbf{V}^{\dagger}$, the UE needs to know $\sigma_{1}, \sigma_{2}, \cdots, \sigma_{r}$ as well as $\mathbf{U}$, to achieve this capacity. The key question is how to communicate the relevant information to the UE. Employing downlink pilots is one such option, but can really be a burden to the UE. Instead, notice that TDD permits another natural feedback mechanism as follows. The BS can perform simple channel inversion using the pseudo-inverse of $\mathbf{H}$, and create low-rate parallel channels to convey the feedback bits. While this inversion technique can be suboptimal from a capacity perspective, the BS can now reliably convey the relevant channel parameters in a small number of transmissions. Selective inversion, after ignoring the low singular values of $\mathbf{H}$, is another tangible technique. Alternately, feedback can be done using the available downlink control channels as well. Since $\mathbf{U}$ has more parameters than $\Sigma$, we will focus on feeding back a quantized version $\hat{\mathbf{U}}$. Once the post-coder is reconstructed at the receiver, simple strategies like energy detection can be used to estimate the effective singular values. For simplicity, we assume in our analysis that $\Sigma$ is conveyed correctly to the receiver or known by other techniques.

Quantization of $\mathbf{U}$ to obtain $\hat{\mathbf{U}}$ results in some loss of information, thereby causing the receiver to not compensate for $\mathbf{U}$ completely. On the other hand, since the quantized feedback necessitates some additional transmissions, it is essential to minimize overheads while maximizing the data rate. We thus explore the trade-off between the overheads for transmitting $\hat{\mathbf{U}}$, and the achievable downlink rates. To this end, we propose efficient quantization schemes for $\mathbf{U}$ under a specified bit constraint, and then attempt to maximize the resulting achievable rate. Under full CSIT and 
the knowledge of $(\hat{\mathbf{U}}, \Sigma)$ at the receiver, the maximum achievable rate can be computed by [12]

$$
\max _{p(\tilde{x} \mid \Sigma, \hat{\mathbf{U}}, \mathbf{V})} I(\tilde{\mathbf{x}} ; y \mid \Sigma, \hat{\mathbf{U}}) \text { s.t. } \mathbb{E}\left[\tilde{\mathbf{x}}^{\dagger} \tilde{\mathbf{x}}\right] \leq P_{T} .
$$

Since full CSIR is not available, it is unclear whether a continuous valued distribution can maximize (3), let alone the Gaussian distribution. In spite of this difficulty, we will show that appropriate Gaussian codebooks perform very well, and achieve rates close to the capacity with full CSI, even while using a moderate number of quantization bits. Notice the difference between conventional fading models where the receiver is typically assumed to have a better version of CSI than that available at the transmitter [12], this adds to the novelty of our analysis. Our numerical results identify good rules of thumb for effectively utilising the feedback bits.

\section{CSI Feedback Scheme and Achievable Rates}

\section{A. Codebook to Compress Unitary Post-coders}

To effectively feedback information about the matrix $\mathbf{U}$ to the UE, a codebook to compress unitary matrices is needed. While several codebook design methods exist, we use the Givens rotation and Householder transformation based decomposition to parameterize the unitary matrix, and compress the parameters. This approach has the advantage that the matrix is represented using scalar angle parameters that are all independent and have well characterized probability distributions [13]. The independence of these parameters converts the compression problem to one where the optimal quantizers for single dimensional random variables needs to be found. These are well known, and thus, this approach is an effective method to quantize the unitary matrices for feedback to the UE.

The Givens rotation and Householder transformations use rotation matrices to null out offdiagonal elements of the unitary matrix. Since rotation matrices do not change the norm of the matrix, the orthogonality of the unitary matrix $\mathbf{U}$ is preserved in the parametrization phase. First, the angles of each element of the first column of the matrix are stored, and then the entries of the column are made real. Subsequently, each of the elements other than the first element are made zero using the appropriate rotation matrix obtained from Givens rotations, and all such angles are stored. These angles form the set of parameters needed for reconstructing the $\mathbf{U}$ matrix. Now, the above two steps are repeated for all the columns in the matrix, making the resulting matrix to be an identity matrix and the corresponding parameters are fed back for reconstruction at the receiver. 
A unitary matrix $\mathbf{U} \in C^{r \times r}$ with orthonormal columns can be decomposed as

$$
\mathbf{U}=\left[\prod_{k=1}^{r-1} \mathbf{D}_{k}\left(\phi_{k, 1}, \cdots, \phi_{k, k}\right) \prod_{l=1}^{r-k} \mathbf{G}_{r-l, r-l+1}\left(\theta_{k, l}\right)\right] \mathbf{D}_{r}\left(\phi_{r, 1}, \ldots \phi_{r, r}\right),
$$

where $\mathbf{D}_{k}\left(\phi_{k, 1}, \cdots, \phi_{k, k}\right)=\operatorname{diag}\left(\mathbf{1}_{r-k},\left\{e^{j \phi_{k, j}}, 1 \leq j \leq k\right\}\right)$ and

$$
\mathbf{G}_{p-1, p}(\theta)=\left[\begin{array}{cccc}
\mathbf{I}_{p-2} & & & \\
& \cos \theta & -\sin \theta & \\
& \sin \theta & \cos \theta & \\
& & & \mathbf{I}_{t-p}
\end{array}\right] .
$$

We note that this is just the transposed representation of the unitary matrix decomposition given in [13]. Here $\mathbf{I}_{q}$ represents the $q \times q$ (block) identity matrix and $\mathbf{1}_{r-k}$ represents a vector of $r-k$ ones. We remark that this expansion is obtained by just taking the transposed form of the expansion in [13]. The matrices $\mathbf{D}_{i}$ and $\mathbf{G}_{j, k}$ contain parameters $\left\{\left(\phi_{k, i}, \theta_{i, l}\right)\right\}$ which, after quantization, are fed back to the receiver. Reconstruction of the matrix is a similar process, in which the operations are performed in reverse. We note that the decomposition can also be performed by performing the operations described above using the rows of the matrix, as opposed to the columns, as we have done in the discussions below.

The number of parameters required to be fed back can be reduced by using the fact that the SVD is not unique. Since the angle information in the left-singular matrix is required for the reconstruction of the unitary matrix at the receiver, both the left and right singular matrices are non-unique [14], and can be replaced with an equivalent pair of unitary matrices. Specifically, for a matrix $\mathbf{H}$ which is of size $r \times t$ and has rank $m$, we have:

$$
\mathbf{H}=\sum_{i=1}^{m} \sigma_{i} \mathbf{u}_{i} \mathbf{v}_{i}^{\dagger}=\sum_{i=1}^{m} \sigma_{i}\left(e^{j \alpha_{i}}\right) \mathbf{u}_{i}\left(e^{j \alpha_{i}} \mathbf{v}_{i}\right)^{\dagger}
$$

where $\mathbf{u}_{i}, \mathbf{v}_{i}$ represent the left and right singular vectors of $\mathbf{H}$ respectively, and the phase factors $\alpha_{i}$ can taken to be any values in $(-\pi, \pi]$ without altering the SVD. Therefore, we can choose $\alpha_{i}$ to make the first row of the unitary matrix $\mathbf{U}$ real, thereby fixing the $m$ parameters to be zero and eliminating the need to feed it back. For an $r \times t$ system having rank $r$, the number of parameters needed to effectively feed back information about the post-coder is $r^{2}-r$. This can be observed for a system having 2 antennas at UE as follows. First, any $2 \times 2$ unitary matrix can be uniquely described by the real parameters $\theta$ and $\phi_{1}, \phi_{2}, \phi_{3}$ as follows [13]:

$$
\mathbf{U}=\underbrace{\left[\begin{array}{cc}
1 & 0 \\
0 & e^{j \phi_{1}}
\end{array}\right]}_{\mathbf{U}_{1}} \underbrace{\left[\begin{array}{cc}
\cos \theta & \sin \theta \\
-\sin \theta & \cos \theta
\end{array}\right]}_{\mathbf{U}_{2}} \underbrace{\left[\begin{array}{cc}
e^{j \phi_{2}} & 0 \\
0 & e^{j \phi_{3}}
\end{array}\right]}_{\mathbf{U}_{3}} .
$$


Notice that

$$
H=\mathbf{U}_{1} \mathbf{U}_{2} \mathbf{U}_{3} \Sigma \mathbf{V}^{\dagger}=\mathbf{U}_{1} \mathbf{U}_{2} \Sigma \mathbf{U}_{3} \mathbf{V}^{\dagger}=\mathbf{U}_{1} \mathbf{U}_{2} \Sigma\left(\mathbf{V} \mathbf{U}_{3}^{\dagger}\right)^{\dagger}
$$

We can observe that matrix $\mathbf{U}_{3}$ is the diagonal matrix $\mathbf{D}_{r}(\cdot)$ in Equation (4). Thus $\mathbf{U}_{1} \mathbf{U}_{2}$ can be taken as the effective post-coder matrix, and $\mathbf{V} \mathbf{U}_{3}^{\dagger}$ becomes the precoder matrix, the latter having no effect on the received signal due to precoding [10]. Thus only $r(r-1)=2$ parameters need to be conveyed for $r=2$. To simplify the parameter representation, notations introduced here for angle parameters (i.e., $\theta$ and $\phi_{j} j=1,2,3$ ) will be used for any further discussions on systems with only 2 antennas at the UE. In general, the effective post-coder matrix to be communicated to the UE can be taken as

$$
\mathbf{U}=\left[\prod_{k=1}^{r-1} \mathbf{D}_{k}\left(\phi_{k, 1}, \cdots, \phi_{k, k}\right) \prod_{l=1}^{r-k} \mathbf{G}_{r-l, r-l+1}\left(\theta_{k, l}\right)\right] \mathbf{I} .
$$

Subsequent to the parameterization of the unitary matrix, we focus on effective quantization of the angle parameters $\left(\phi_{i, j}, \theta_{l, m}\right)$. We use individual scalar quantizers for each independent parameter. Since $\phi_{i, j} 1 \leq j \leq r, j \leq i \leq r$ are distributed uniformly in $(-\pi, \pi]$, uniform quantizers are suitable. The values $\theta_{i, l} \forall i=\{1, \ldots, r-1\}, l=\{1, \ldots, r-i\}$ have the probability density

$$
p\left(\theta_{i, l}\right)=2 l\left(\sin \theta_{i, l}\right)^{(2 l-1)} \cos \theta_{i, l}, 0 \leq \theta_{i, l}<\frac{\pi}{2},
$$

which only depends on the index $l$. The optimal scalar quantizer given by the Lloyd's algorithm [15] can now be used to quantize each of these parameters. Let $\epsilon_{\theta_{l}}^{2}$ be the Mean Squared Error (MSE) of the scalar quantizer for the parameter $\theta_{i, l}$ in (7). The MSE between the post-coder matrix $\mathbf{U}$ and its quantization $\hat{\mathbf{U}}$, denoted as $\epsilon_{\text {Lloyd }}^{2}$, can be computed as

$$
\epsilon_{\text {Lloyd }}^{2}=\mathbb{E}\|\mathbf{U}-\hat{\mathbf{U}}\|_{F}^{2}=\mathbb{E}\left[\operatorname{Tr}\left(\hat{\mathbf{U}}^{\dagger} \mathbf{U}-\mathbf{I}_{r}\right)^{\dagger}\left(\hat{\mathbf{U}}^{\dagger} \mathbf{U}-\mathbf{I}_{r}\right)\right]
$$

where $\|\mathbf{A}\|_{F}$ denotes the Frobenius norm.

Theorem 1. For the post-coder matrix $\mathbf{U}$ represented by (4), let $\hat{\mathbf{U}}$ be the unitary matrix reconstructed from the quantized values of $\phi_{i, j}$ and $\theta_{i, j}$ obtained as per the Lloyd's algorithm, with $b_{1}$ bits used to quantize each $\phi_{i, j}$. Then the quantization MSE satisfies

$$
\epsilon_{\text {Lloyd }}^{2} \leq r(r-1)\left(1-\operatorname{sinc}\left(2^{-b_{1}}\right)\right)+\sum_{l=1}^{r-1} 2(r-l) \epsilon_{\theta_{l}}^{2} .
$$

Proof. The proof can be found in Appendix A. 
While the MSE $\epsilon_{\theta_{l}}^{2}$ in quantizing $\theta_{i, l}$ can be evaluated numerically using (7), efficient closed form approximations are also available. In particular, the results in [16] suggest that using $b_{2}$ bits for quantization will yield an MSE

$$
\epsilon_{\theta_{l}}^{2} \approx \frac{1}{12\left(2^{2 b_{2}}\right)}\left(\int_{0}^{\frac{\pi}{2}} p\left(\theta_{k, l}\right)^{\frac{1}{3}} \mathrm{~d} \theta_{k, l}\right)^{3}
$$

Using (7) in the above expression, we get

$$
\epsilon_{\theta_{l}}^{2} \approx \frac{\beta\left(\frac{2}{3}, \frac{l+1}{3}\right)^{3} l}{12\left(2^{2 b_{2}+2}\right)}
$$

where $\beta(x, y), x \in \mathbb{R}^{+}, y \in \mathbb{R}^{+}$is the beta function defined by [17],

$$
\beta(x, y)=\int_{0}^{1} t^{x-1}(1-t)^{y-1} \mathrm{~d} t .
$$

Using (9) and (10)

$$
\epsilon_{\text {Lloyd }}^{2} \approx r(r-1)\left(1-\operatorname{sinc}\left(2^{-b_{1}}\right)\right)+\sum_{l=1}^{r-1} \frac{\beta\left(\frac{2}{3}, \frac{l+1}{3}\right)^{3} l(r-l)}{3\left(2^{2 b_{2}(l)+3}\right)},
$$

where $b_{2}(l)$ bits were used to quantize the parameter $\theta_{i, l}$ as per the Lloyd's algorithm. Clearly the Lloyd's algorithm achieves an exponential decay of MSE as more quantization bits are employed for each scalar parameter. This is also evident in $(11)$ as the functions $1-\operatorname{sinc}\left(2^{-b}\right)$ as well as $\frac{\beta\left(\frac{2}{3}, \frac{l+1}{3}\right)^{3} l(r-l)}{3\left(2^{2 b+3}\right)}$ decrease as $O\left(2^{-2 b}\right)$. While (11) was introduced as an approximate bound along the lines of [16], numerical results show that the RHS indeed gives a close upperbound to the MSE, as shown in Figure 2.

Thus, given a total bit budget of $b$ bits, we can allocate $b_{1}$ bits for quantizing each $\phi_{i}$, and $b_{2}(l)$ bits to each $\theta_{i, l}$, in such a way that the MSE in (11) is minimized. By relaxing the integer constraints, straightforward solutions are possible for this minimization.

For particular configurations, the bound from Theorem 1 can be made even more tight. For instance, the following theorem computes the exact MSE when $r=2$.

Theorem 2. For a $2 \times 2$ MIMO system, let the unitary post-coder matrix $\mathbf{U}$ be parameterized by $\phi_{1}$ and $\theta$. Let each of these parameters be quantized using b bits, and $\hat{\mathbf{U}}$ be the post-coder reconstruction from the quantized values. Then,

$$
\epsilon_{\text {Lloyd }}^{2}=4-2 \mathbb{E}[\cos (\theta-\hat{\theta})]\left(1+\operatorname{sinc}\left(2^{-b}\right)\right)
$$

where $\hat{\theta}$ is the quantized value of $\theta$.

Proof. The proof is given in Appendix B. 


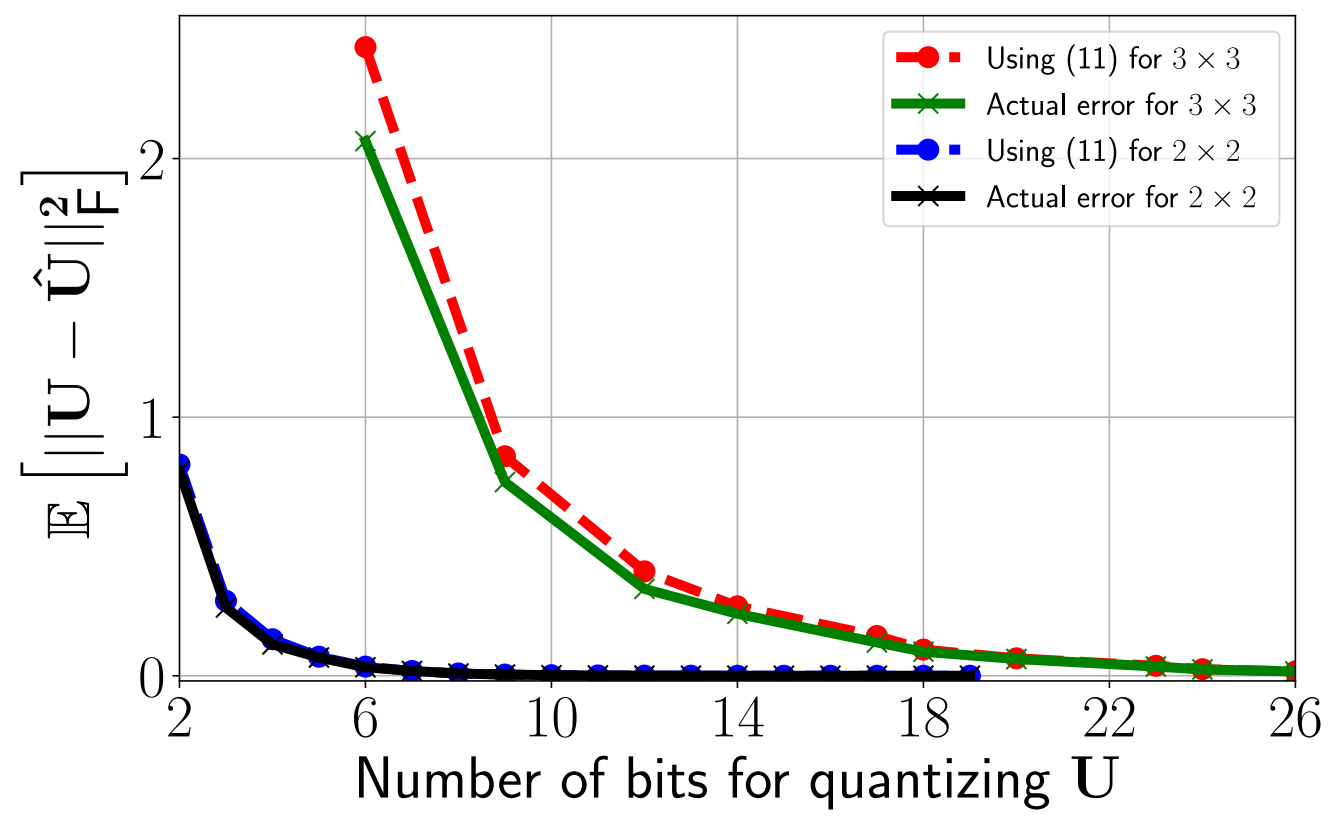

Fig. 2: Average post-coder quantization error: Actual Vs Equation (11).

Our main objective now is to characterize the ergodic downlink rates under quantized postcoder feedback to the receiver, in terms of the MSE $\epsilon_{\text {Lloyd }}^{2}$.

\section{B. Power Allocation and Ergodic Achievable Rates}

To obtain the ergodic capacity of the system, it is necessary to allocate power in an optimal manner across blocks, based on the distribution of the channel and its current realization, while maintaining a long term average power of $P_{T}$. Notice that we assumed $r \leq t$, i.e. the number of antennas at the $\mathrm{UE} r$ is at most that at the BS, which has $t$ antennas. Let us start by recollecting the optimal power control law when there is full CSI at the transmitter as well as receiver, this is based on the singular values of the fading matrix [10]. The pdf of the unordered singular values of $\mathbf{H H}^{\dagger}$ is given by [18]:

$$
g_{r, t}(\sigma)=\frac{1}{r} \sum_{k=0}^{r-1} \frac{k !}{(k+t-r) !}\left[L_{k}^{t-r}(\sigma)\right]^{2} \sigma^{t-r} e^{-\sigma},
$$

where $L(\sigma)$ is the Laguerre polynomial given by

$$
L_{k}^{n}(\sigma)=\frac{e^{\sigma}}{k ! \sigma^{n}} \frac{d^{k}}{d \sigma^{k}}\left(e^{-\sigma} \sigma^{n+k}\right) .
$$

The optimal power control is then given by the celebrated water-filling law [12], which appropriately allocates power over the $r$ parallel channels (one corresponding to each singular value) in each block, and across blocks as well. More specifically, a positive parameter $\lambda$ such that

$$
\int_{\lambda}^{\infty}\left(\frac{1}{\lambda}-\frac{1}{\sigma^{2}}\right) g_{r, t}(\sigma) d \sigma=\frac{P_{T}}{r}
$$


is chosen, and $P(\sigma)=\max \left\{0,\left(\lambda^{-1}-\sigma^{-2}\right)\right\}$ is the power allocated to the channel with singular value $\sigma$. The ergodic rate achievable with full CSI is then [12]

$$
\mathrm{C}_{\text {full CSI }}=\log \left(r \int_{\lambda}^{\infty} \frac{\sigma^{2}}{\lambda} g_{r, t}(\sigma)\right) d \sigma .
$$

For future use, let us denote the average received power in each of the $r$ parallel channels as

$$
P_{\mathrm{rx}}=\int_{\lambda}^{\infty}\left(\frac{\sigma^{2}}{\lambda}-1\right) g_{r, t}(\sigma) d \sigma .
$$

We now characterize the gap between the achievable rates obtained by the proposed quantization scheme, and the capacity evaluated by (3).

\section{Gap to Ergodic Capacity}

It turns out that the proposed communication scheme under quantized feedback can achieve rates close to (14) itself, when a sufficient number of quantization bits are available. The following theorem characterizes the rate gap to $\mathrm{C}_{\text {full }} \mathrm{CSI}$ in terms of the quantization MSE.

Theorem 3. For a MIMO channel matrix $\mathbf{H}$ with i.i.d Rayleigh entries, the proposed quantization scheme can achieve a rate of

$$
\max _{p(x \mid \Sigma, \hat{\mathbf{U}})} I(\mathbf{x} ; \mathbf{y} \mid \Sigma, \hat{\mathbf{U}}) \geq C_{\text {full CSI }}-r \log _{2}\left(1+\frac{P_{\mathrm{rx}}}{r} \epsilon_{\text {Lloyd }}^{2}\right),
$$

where $\epsilon_{\mathrm{Lloyd}}^{2}$ and $P_{r x}$ are given in (8) and (15) respectively, and $\Sigma=\operatorname{diag}\left(\sigma_{1}, \cdots, \sigma_{r}\right)$.

Proof. The proof is relegated to Appendix C.

While Theorem 3 provides a convenient lower bound on the achievable rate for the downlink system, an astute reader might have observed that (38) can give even tighter bounds. In any case,

since the MSE $\epsilon_{\text {Lloyd }}^{2}$ decreases exponentially fast as more and more bits are used for quantization, the average rates quickly reach $\mathrm{C}_{\text {full CSI }}$.

\section{Massive MIMO Systems}

While the techniques suggested so far enable us to achieve full CSI capacity as finer quantizations of the post-coder become available, they do not explicitly take into account the effect of channel hardening present in massive MIMO systems. More specifically, for a fixed number of UE antennas, the singular values and singular vectors of the channel hardens or concentrates as the number $t$ of antennas at the BS increases [1, 19]. While channel hardening can lead to better quantizers having lower mean square error, this may not always translate to an increase in 
achievable rates. The major reason is that in order to reap the benefits of a significant boost in receive signal strength achieved with massive MIMO, a post-coder matrix without any mismatch at the receiver is required. Thus achieving rates approaching the full CSI capacity will happen at the expense of more quantization bits. However, if a constant gap to capacity is admissible, then the burden on feedback can be considerably reduced. In particular, the transmitter can precode to avoid any receiver mismatch, thereby avoiding the post-coder requirement. This is particularly appealing for massive MIMO, as the gap to full CSI capacity is a small constant then.

Our communication scheme works as follows. Consider $r$ code-books of equal rate, where the entries of each codebook are generated according to unit variance zero mean Gaussian distribution. Thus $r$ transmitted data symbols at each instant is denoted by the vector $\mathbf{d}$. In order to convey $\mathbf{d}$, the transmitter sends $\tilde{x}=\sqrt{\alpha(\Sigma)} \mathbf{H}^{\dagger}\left(\mathbf{H H}^{\dagger}\right)^{-1} \mathbf{d}$ in $(1)$, where $\sqrt{\alpha(\Sigma)}$ is a positive real number that depends on $\Sigma$. This translates to pre-multiplying the transmitted signal by the scaled pseudoinverse of the channel. Notice that this is not same as channel inversion, as the non-negative scaling factor $\alpha(\Sigma)$ is a function of the singular values of $\mathbf{H}$, and this is crucial to our scheme. The receiver obtains

$$
\mathbf{y}=\sqrt{\alpha(\Sigma)} \mathbf{H H}^{\dagger}\left(\mathbf{H H}^{\dagger}\right)^{-1} \mathbf{d}+\eta=\sqrt{\alpha(\Sigma)} \mathbf{d}+\eta
$$

where the choice of $\alpha(\Sigma)$ ensures that the average power constraint is satisfied. W.l.o.g assume that $\eta \sim \mathcal{N} C(0, \mathbb{I})$. The constraint $\mathbb{E}\left[\operatorname{Tr}\left(\mathbf{x x}^{\dagger}\right)\right] \leq P_{T}$ will imply that

$$
\mathbb{E}\left[\alpha(\Sigma) \operatorname{Tr}\left(\Sigma^{-2}\right)\right] \leq P_{T}
$$

The choice of $\alpha(\Sigma)$ can now be made to maximize the resulting achievable rate. Using the Lagrange multiplier $\lambda$, one can optimize by applying KKT conditions on the unconstrained cost function

$$
R(\alpha(\Sigma), \lambda)=r \mathbb{E}[\log (1+\alpha(\Sigma))]-\lambda\left(\mathbb{E}\left[\alpha(\Sigma) \operatorname{Tr}\left(\Sigma^{-2}\right)\right]-P_{T}\right)
$$

Differentiating with respect to $\alpha(\Sigma)$ for each channel realization, and equating to zero, we get

$$
\frac{r}{1+\alpha(\Sigma)}=\lambda \operatorname{Tr}\left(\Sigma^{-2}\right)
$$

Incorporating this back into the constraint and eliminating $\lambda$, the rate expression simplifies to

$$
R_{Z F}=r \mathbb{E}\left[\log \left(\frac{P_{T}+\mathbb{E}\left[\operatorname{Tr}\left(\Sigma^{-2}\right)\right]}{\operatorname{Tr}\left(\Sigma^{-2}\right)}\right)\right] .
$$

Remarkably, this rate is achieved without any CSI feedback to the receiver at all. We will show that this scheme performs reasonably well, albeit with a small gap to capacity, when there are hundreds of transmit antennas at the BS. We call this the ZF precoding approach. 
For the particular case where the number of transmit and receive antennas are the same, i.e. $r=t$, the above optimization does not yield a bounded solution. In this situation, we selectively invert the channel to transmit data only one stream of data that corresponds to the largest singular value.

\section{Simulation Results}

We now present simulation results that characterize the downlink rate based on the amount of feedback used to represent the post-coder $\mathbf{U}$ at the receiver. We consider situations where the BS has several antennas and a UE with 2 or 3 receive antennas. Quantized post-coder feedback is beneficial here to approach the full CSI capacity. Finally, we comment on the performance of precoding in the case of massive MIMO systems.

\section{A. Quantization of unitary post-coder}

The first step in MIMO systems with quantized feedback is to have codebooks for the unitary post-coder matrix. More specifically, $\theta_{i, j}$ in (4) is quantized as described in Section III-A, using the conventional Lloyd's algorithm [15]. Since the distribution of each $\phi_{i, j}$ is uniform, a uniform quantizer was used for these parameters. Given a total feedback bit budget, a bit allocation maximizing the rate bound of (38) is used for each plot using our quantization scheme. As an example, for a $2 \times 2$ post-coder matrix $\mathbf{U}$, we need to quantize two phase parameters, say $\theta$ and $\phi$. If $b$ bits are available, we find the best combination of $b_{1}$ bits to quantize $\phi$ and $b_{2}$ bits to quantize $\theta$ such that the maximum of (38) is obtained and plotted in Fig. 2. The reconstructed matrix obtained from the quantized parameters, viz. $\hat{\phi}$ and $\hat{\theta}$ is denoted as $\hat{\mathbf{U}}$. The quantization error is now measured as $\mathbb{E}\left[\|\mathbf{U}-\hat{\mathbf{U}}\|_{F}^{2}\right]$. The mean squared error in reconstruction is illustrated in Fig. 2 for 2 and 3 antennas at the UE, as a function of the total number of quantization bits used to represent $\hat{\mathbf{U}}$. Observe that about a dozen bits can make the MSE sufficiently small for systems with 2 antennas at UE.

\section{B. Downlink Data Rates with post-coder feedback}

We now study how the quantization of $\mathbf{U}$ impacts the achieved rate. As discussed in Section III-D the post-coder approach is expected to be beneficial when the number of antennas at the $\mathrm{BS}$ is not very large, whereas the ZF precoding approach can be useful for massive MIMO systems where the number of transmit antennas $(t)$ far exceeds that at the UE $(r)$. However, observe that the optimization specified in (19) as such has no solution for $t=r$. 


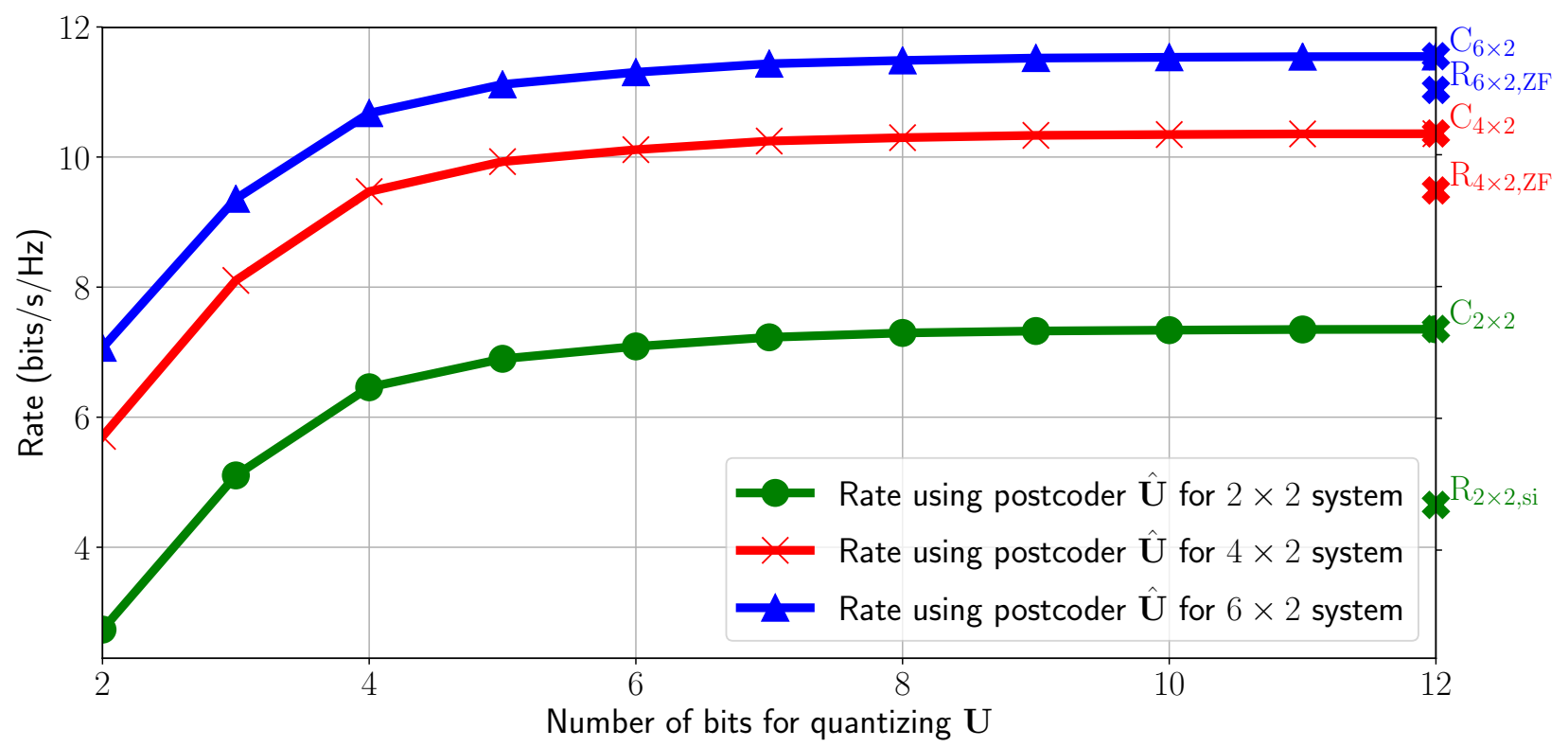

Fig. 3: Lower bound on achievable rate when optimal number of bits are allocated to $\phi_{3}$ and $\theta$ compared with rate achieved using zero forcing precoding for $6 \times 2\left(\mathrm{R}_{6 \times 2, \mathrm{ZF}}\right), 4 \times 2\left(\mathrm{R}_{4 \times 2, \mathrm{ZF}}\right)$ and $2 \times 2\left(\mathrm{R}_{2 \times 2, Z \mathrm{ZF}}\right)$ systems, where $\mathrm{C}_{6 \times 2}, \mathrm{C}_{4 \times 2}$ and $\mathrm{C}_{2 \times 2}$ are the respective system capacities at $10 \mathrm{~dB}$ and $\mathrm{R}_{2 \times 2 \text {,si }}$ is the rate achieved using only the best Eigen mode for a $2 \times 2$ system.

For systems with 2 antenna UEs, from Fig. 3, we see that the achievable rate is almost the downlink capacity (i.e. when perfect CSI is available) if about 10 bits are used for quantization of the parameters $\phi, \theta$ for the $2 \times 2$ post-coder matrix. More significantly, when compared to the ZF precoding based approach, we find that the significant gains in rate can be observed when the post-coder is used with 4 or more bits of quantization. In the $2 \times 2$ case, since the optimization to perform transmit domain ZF precoding does not yield a feasible solution, we selectively invert only the best channel as a comparison with the feedback based approach. We find that even with just 3 bits of quantization, the performance using feedback far exceeds that achievable using purely transmitter compensation, thereby justifying the use of post-coder feedback.

For systems with 3 antennas at the UE, the performance trends are similar to those of systems with 2 antennas, except that it requires a larger number of bits to achieve rates close to the upperbound, as seen in Fig. 4. From Observe that the effect of quantization becomes small after 24 bits in Fig. 2. This manifests as enhanced achievable rates in Fig. 4. For the $3 \times 3$ case, we also find that selectively inverting to use only the best channel yields a much poorer performance. Clearly, post-coder feedback is always better in these systems.

The variation of the achievable rate with SNR for a $4 \times 2$ MIMO system is shown in Fig. 5, 


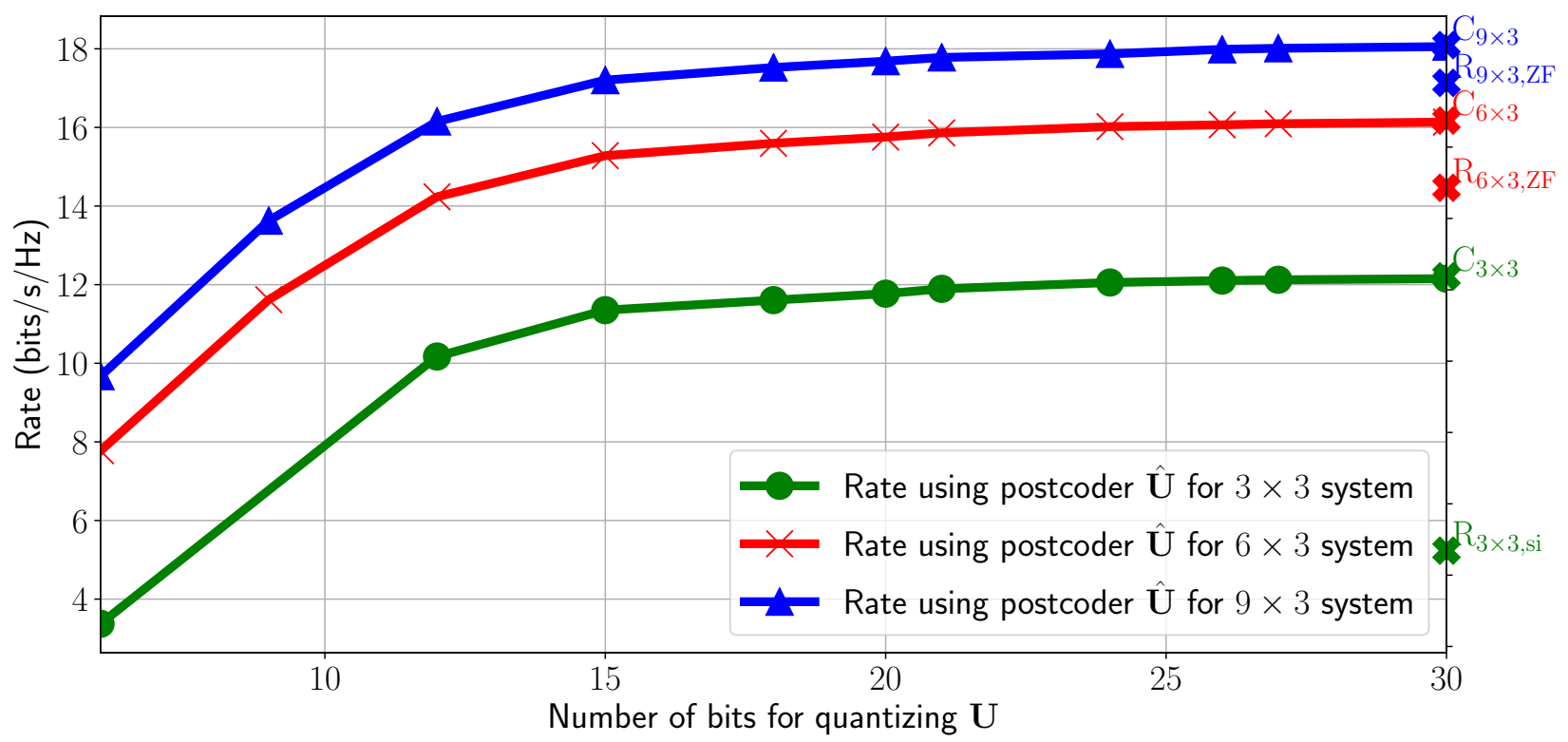

Fig. 4: Lower bound on achievable rate when optimal number of bits are allocated to $\phi_{2,1}$, $\phi_{3,1}, \phi_{3,2}$ and $\theta_{2,1}, \theta_{3,1}, \theta_{3,2}$ compared with rate achieved using zero forcing precoding for $9 \times 3$ $\left(\mathrm{R}_{9 \times 3, \mathrm{ZF}}\right), 6 \times 3\left(\mathrm{R}_{6 \times 3, \mathrm{ZF}}\right)$ and $3 \times 3\left(\mathrm{R}_{3 \times 3, \mathrm{ZF}}\right)$ systems, where $\mathrm{C}_{9 \times 3}, \mathrm{C}_{6 \times 3}$ and $\mathrm{C}_{3 \times 3}$ are the respective system capacities at $10 \mathrm{~dB}$ and $\mathrm{R}_{3 \times 3 \text {,si }}$ is obtained by using only the best eigen mode in a $3 \times 3$ system.

where the ratio of the achievable rate using $\hat{\mathbf{U}}$ against the downlink capacity is shown as a percentage. Observe that, as the SNR increases, achieving a rate close to capacity requires a larger number of bits. This is mainly because efficient allocation of resources at high SNR requires a more accurate $\hat{\mathbf{U}}$ at the receiver.

\section{Effective utilization of bits alloted}

As discussed in the previous section, when the quantization bit budget is low, allotting bits to all the singular vectors of the channel may not be effective for systems having more than 3 antennas at UE, since inaccurate quantization of singular vectors would reduce the rate significantly. An alternate approach is to allocate all power to a single singular vector and quantize only that, since the benefit from more accurate quantization could outweigh the benefit from using all channels in this situation. Therefore, we also study the effect of quantizing just one singular vector, rather than the full matrix. Notice that our quantization scheme using Givens rotation cannot directly yield individual quantizers for each of the singular vectors. We, therefore, parameterize only the best singular vector with all available bits to study the performance, as described in Section IV-B. 


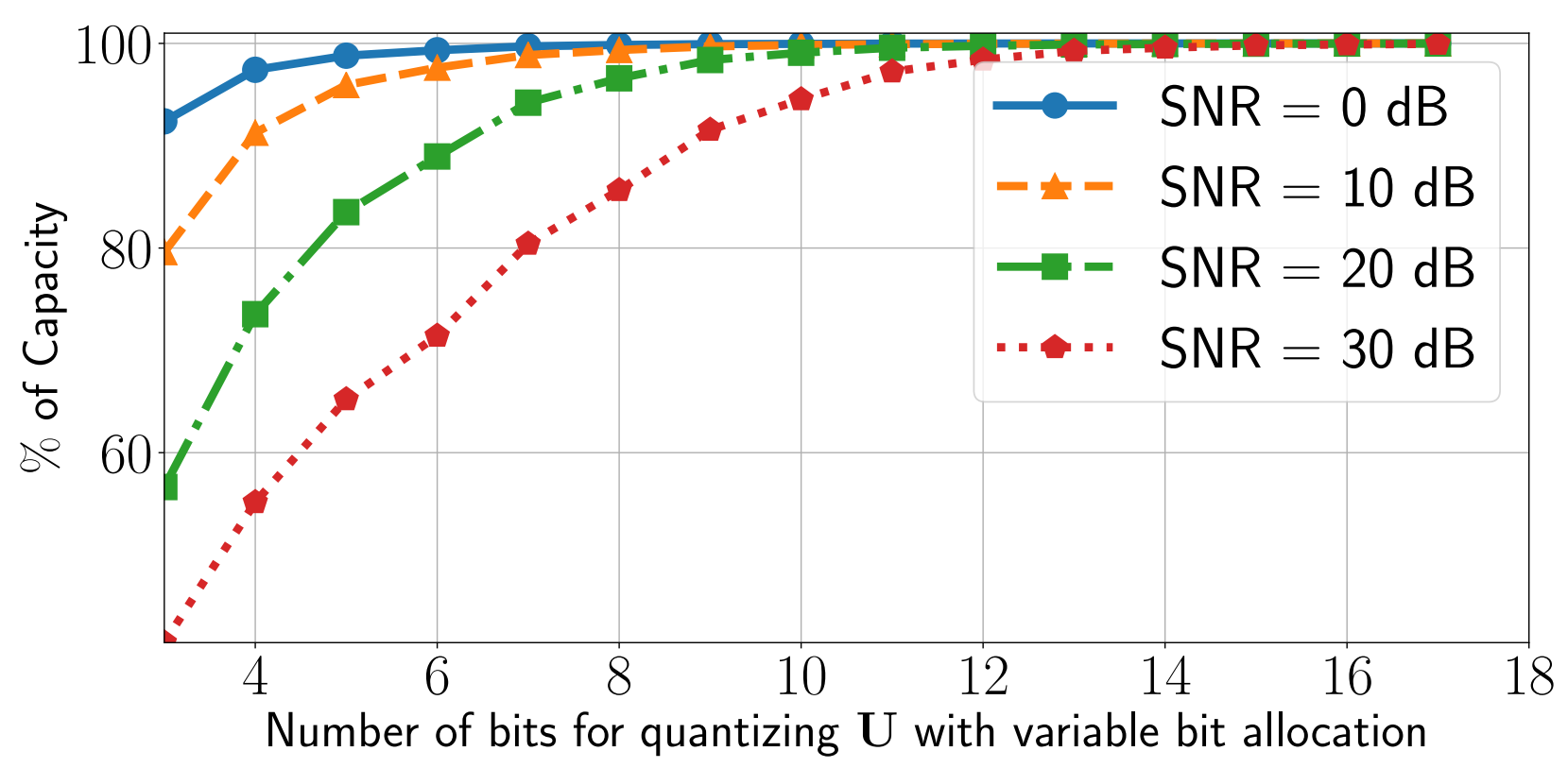

Fig. 5: Percentage of capacity achieved for a $4 \times 2$ MIMO system at different SNRs.

In Fig. 6 we see that, when using fewer than 8 bits for quantizing the post-coder $\mathbf{U}$, the rate achieved using a single channel is higher than the rate achieved using three channels. This is because, for one channel, the number of parameters required to be fed back are just 4 i.e, $\phi_{2,2}$, $\phi_{2,3}, \theta_{1,1}$ and $\theta_{1,2}$, whereas 6 parameters are needed for the full matrix, viz. $\phi_{2,2}, \phi_{2,3}, \phi_{3,3}$, $\theta_{1,1}, \theta_{1,2}$ and $\theta_{2,1}$. Therefore, when the number bits is small, quantizing only the best singular vector's parameters results in more useful CSI than when quantizing all channel parameters simultaneously with the same bit budget. This benefit diminishes as the number of bits used for quantization increases, and the rate achieved using one channel saturates, while using three channels yields better performance.

\section{Bit error rate}

In addition to high achievable rates, we now show that our communication schemes maintain a low BER on each parallel eigen mode of the MIMO channel. Thus the adverse effects due to the inaccurate post-coder are kept low. In Fig. 7, we observe that the BER obtained when transmitting a QPSK signal over the spatial channel that corresponds to the larger singular value of a $4 \times 2$ MIMO system with post-coder feedback is sensitive to the number of bits used to quantize $\mathbf{U}$. We see that the use of fewer than 10 bits results in poor performance, since the $\hat{\mathbf{U}}$ at the receiver is not sufficiently accurate to diagonalize the channel. However, with 12 bits, the performance is close to that obtained using the perfect post-coder. 


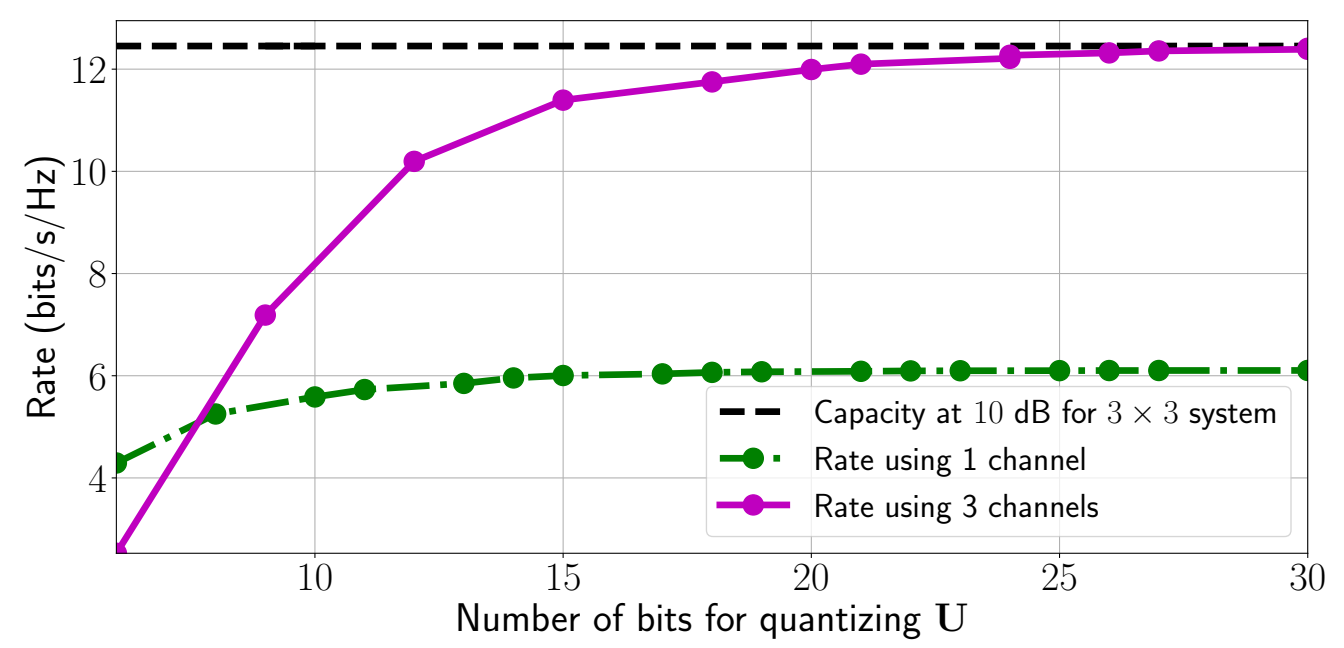

Fig. 6: Rate achieved vs channels used, for $3 \times 3$ system.

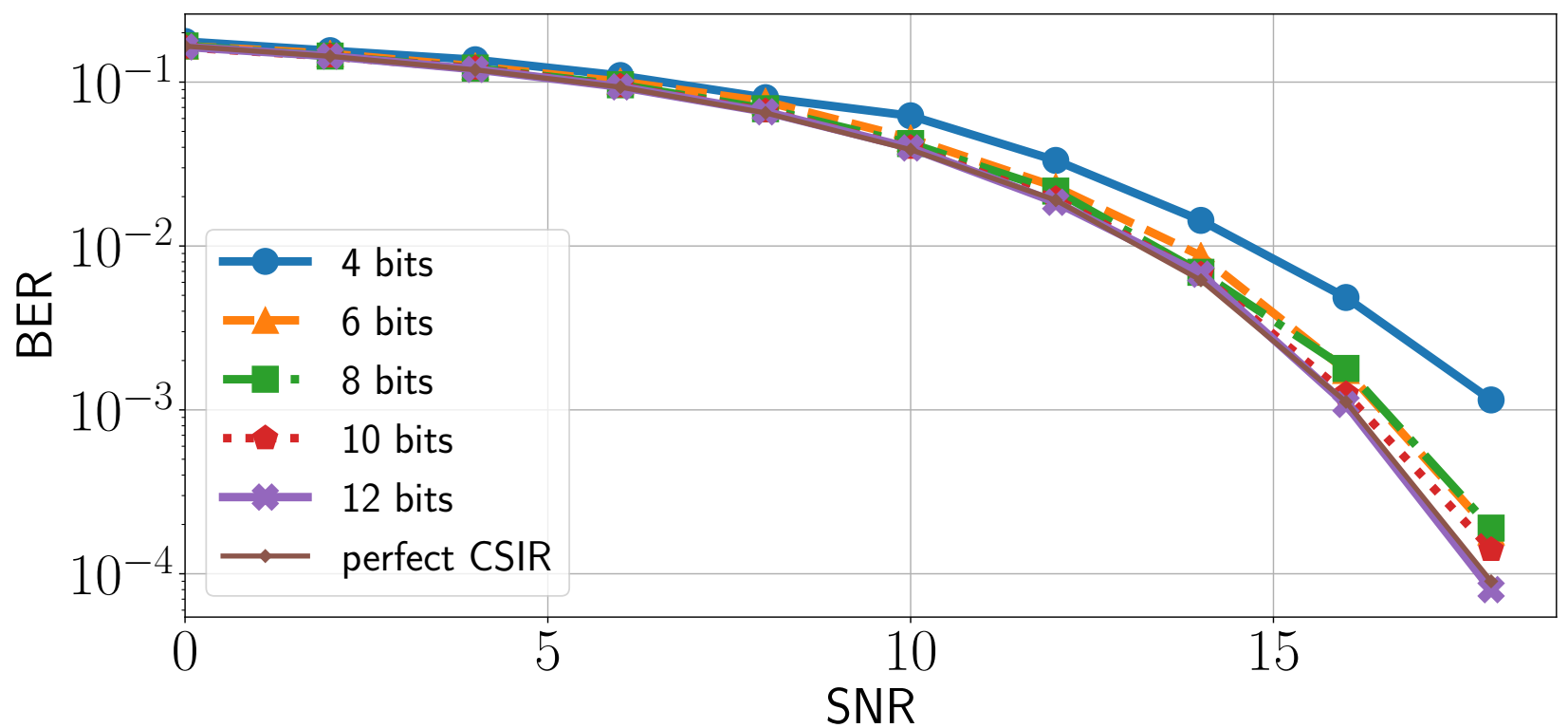

Fig. 7: BER of QPSK with variable bit allocation for $\theta$ and $\phi$, of a $4 \times 2$ system at $10 \mathrm{~dB}$.

Although we considered the case where the post-coder is fully estimated only at the BS and fed back to the UE, one could also estimate the post-coders via downlink pilots at the UE. However, in this case, refining the estimate at the UE may require more resources. We further remark that an additional reduction in the quantization bit requirement is feasible by adaptive quantization approaches, particularly for slowly varying channels, as described in [13]. For example, using just 1 bit per parameter with an adaptive tracking mechanism may further reduce the bit budget. The convergence of $\hat{\mathbf{U}}$ to $\mathbf{U}$ is still governed by the analysis presented here. 


\section{Conclusion}

We have considered downlink transmission in MIMO-TDD systems where UEs possess multiple antennas, where post-coder information is used to enhance performance. Achieving the capacity and enabling channel parallelization requires that the receiver know the right singular vectors of the MIMO channel matrix. We take the approach of using the Givens rotations and Householder transformations to parameterize the unitary post-coders, which permits us to represent unitary matrices in terms of independent scalar parameters that represent rotation angles. When the UE has 2 antennas, we show that about 8 bits suffice to quantize the post-coder accurately. This requirement can be reduced to less than 2 bits per training instant when adaptive tracking of the parameters is employed. When the UE has 3 antennas 26 bits are sufficient to accurately represent the post-coder, with adaptive refinement requiring only 6 bits per instant. We further show that, when fewer bits are available, quantizing the dominant singular vector alone achieves higher rates than quantizing and feeding back the complete post-coder. Our simulations reveal that the proposed quantization approach yields performance close to that achieved using the accurate post-coder, both in terms of being close to the link capacity as well as achieving low BER. Future work would focus on extending these techniques to UEs to multi-user scenarios where post-coder feedback can be used for eliminating interference and aligning transmissions for various users.

\section{REFERENCES}

[1] E. G. Larsson, O. Edfors, F. Tufvesson, and T. L. Marzetta, "Massive MIMO for next generation wireless systems," IEEE Communications Magazine, vol. 52, no. 2, pp. 186-195, 2014.

[2] D. J. Love, R. W. Heath, V. K. Lau, D. Gesbert, B. D. Rao, and M. Andrews, "An overview of limited feedback in wireless communication systems," IEEE Journal on Selected Areas in Communications, vol. 26, no. 8, pp. 1341-1365, 2008.

[3] S. N. Donthi and N. B. Mehta, "Joint performance analysis of channel quality indicator feedback schemes and frequencydomain scheduling for LTE," IEEE Transactions on Vehicular Technology, vol. 60, no. 7, pp. 3096-3109, 2011.

[4] D. J. Love and R. W. Heath, "Limited feedback precoding for spatial multiplexing systems," in GLOBECOM'O3. IEEE Global Telecommunications Conference (IEEE Cat. No. 03CH37489), vol. 4. IEEE, 2003, pp. 1857-1861.

[5] S. Schwarz and M. Rupp, "Predictive quantization on the stiefel manifold," IEEE Signal Processing Letters, vol. 22, no. 2, pp. 234-238, 2014.

[6] J. Choi, B. Mondal, and R. W. Heath, "Interpolation based unitary precoding for spatial multiplexing mimo-ofdm with limited feedback," IEEE Transactions on Signal Processing, vol. 54, no. 12, pp. 4730-4740, 2006.

[7] June Chul Roh and B. D. Rao, “Channel feedback quantization methods for miso and mimo systems," in 2004 IEEE 15th International Symposium on Personal, Indoor and Mobile Radio Communications (IEEE Cat. No.04TH8754), vol. 2, Sep. 2004, pp. 805-809 Vol.2.

[8] J. Kim and I. Lee, "802.11 WLAN: history and new enabling MIMO techniques for next generation standards," IEEE Communications Magazine, vol. 53, no. 3, pp. 134-140, 2015. 
[9] J. C. Roh and B. D. Rao, “An efficient feedback method for MIMO systems with slowly time-varying channels," in WCNC, vol. 2. IEEE, 2004, pp. 760-764.

[10] E. Telatar, "Capacity of multi-antenna Gaussian channels," European Transactions on Telecommunications, vol. 10, no. 6, pp. 585-595, 1999.

[11] A. Goldsmith, Wireless Communications. Cambridge University Press, 2005.

[12] D. Tse and P. Viswanath, Fundamentals of Wireless Communication. Cambridge University Press, 2005.

[13] J. C. Roh and B. D. Rao, "Efficient feedback methods for MIMO channels based on parameterization," IEEE Transactions on Wireless Communications, vol. 6, no. 1, pp. 282-292, 2007.

[14] L. N. Trefethen and D. Bau III, Numerical Linear Algebra. SIAM, 1997, vol. 50.

[15] A. Gersho and R. M. Gray, Vector quantization and signal compression. Springer Science \& Business Media, 2012 , vol. 159.

[16] N. Judell and L. Scharf, "A simple derivation of Lloyd's classical result for the optimum scalar quantizer (Corresp.)," IEEE Transactions on Information Theory, vol. 32, no. 2, pp. 326-328, March 1986.

[17] M. Abramowitz and I. A. Stegun, Handbook of mathematical functions with formulas, graphs, and mathematical tables. US Government printing office, 1948, vol. 55.

[18] A. M. Tulino, S. Verdú et al., "Random matrix theory and wireless communications," Foundations and Trends® in Communications and Information Theory, vol. 1, no. 1, pp. 1-182, 2004.

[19] B. M. Hochwald, T. L. Marzetta, and V. Tarokh, "Multiple-antenna channel hardening and its implications for rate feedback and scheduling," IEEE Trans. Inf. Theory, vol. 50, no. 9, pp. 1893-1909, 2004.

[20] D. J. Garling, Inequalities: a journey into linear analysis. Cambridge University Press, 2007.

\section{Appendix A}

\section{Proof of Theorem 1}

Denoting $\mathbf{D}_{k}\left(\phi_{k, 1}, \ldots, \phi_{k, k}\right)$ in (6) as $\mathbf{D}_{k}$, and $\mathbf{G}_{r-l, r-l+1}\left(\theta_{k, l}\right)$ as $\mathbf{G}_{r-l}^{k, l}$, we have

$$
\mathbf{U}=\left[\prod_{k=1}^{r-1} \mathbf{D}_{k} \prod_{l=1}^{r-k} \mathbf{G}_{r-l}^{k, l}\right] \mathbf{I} .
$$

Similarly, the quantized post-coder matrix $\hat{\mathbf{U}}$ is given as

$$
\hat{\mathbf{U}}=\left[\prod_{k=1}^{r-1} \hat{\mathbf{D}}_{k} \prod_{l=1}^{r-k} \hat{\mathbf{G}}_{r-l}^{k, l}\right] \mathbf{I},
$$

where $\mathbf{D}_{k}\left(\hat{\phi}_{k, 1}, \cdots, \hat{\phi}_{k, k}\right)=\hat{\mathbf{D}}_{k}$ and $\mathbf{G}_{r-l, r-l+1}\left(\hat{\theta}_{r, l}\right)=\hat{\mathbf{G}}_{r-l}^{k, l}$. Letting $\mathbf{Q}=\left(\hat{\mathbf{U}}^{\dagger} \mathbf{U}-\mathbf{I}_{r}\right)^{\dagger}\left(\hat{\mathbf{U}}^{\dagger} \mathbf{U}-\mathbf{I}_{r}\right)$,

$$
\operatorname{Tr}(\mathbf{Q})=\|\mathbf{U}-\hat{\mathbf{U}}\|_{F}^{2}=\left\|\left[\prod_{k=1}^{r-1} \mathbf{D}_{k} \prod_{l=1}^{r-k} \mathbf{G}_{r-l}^{k, l}\right]-\left[\prod_{k=1}^{r-1} \hat{\mathbf{D}}_{k} \prod_{l=1}^{r-k} \hat{\mathbf{G}}_{r-l}^{k, l}\right]\right\|_{F}^{2}
$$


Taking $\mathbf{E}_{1}=\mathbf{D}_{1}-\hat{\mathbf{D}}_{1}$, and applying triangle inequality on the above expression

$$
\begin{aligned}
\operatorname{Tr}(\mathbf{Q}) & \leq\left\|\left[\prod_{l=1}^{r-1} \mathbf{G}_{r-l}^{1, l} \prod_{k=2}^{r-1} \mathbf{D}_{k} \prod_{l=1}^{r-k} \mathbf{G}_{r-l}^{k, l}\right]-\left[\prod_{l=1}^{r-1} \hat{\mathbf{G}}_{r-l}^{1, l} \prod_{k=2}^{r-1} \hat{\mathbf{D}}_{k} \prod_{l=1}^{r-k} \hat{\mathbf{G}}_{r-l}^{k, l}\right]\right\|_{F}^{2}+\left\|\mathbf{E}_{1} \mathbf{D}_{1} \hat{\mathbf{D}}_{1}\right\|_{F}^{2} \\
& =\left\|\left[\prod_{l=1}^{r-1} \mathbf{G}_{r-l}^{1, l} \prod_{k=2}^{r-1} \mathbf{D}_{k} \prod_{l=1}^{r-k} \mathbf{G}_{r-l}^{k, l}\right]-\left[\prod_{l=1}^{r-1} \hat{\mathbf{G}}_{r-l}^{1, l} \prod_{k=2}^{r-1} \hat{\mathbf{D}}_{k} \prod_{l=1}^{r-k} \hat{\mathbf{G}}_{r-l}^{k, l}\right]\right\|_{F}^{2}+\left\|\mathbf{E}_{1}\right\|_{F}^{2} .
\end{aligned}
$$

Now, taking $\mathbf{E}_{1, r-1}^{\prime}=\mathbf{G}_{1}^{1, r-1}-\hat{\mathbf{G}}_{1}^{1, r-1}$,

$$
\begin{aligned}
\operatorname{Tr}(\mathbf{Q}) & \leq\left\|\left[\prod_{l=1}^{r-2} \mathbf{G}_{r-l}^{1, l} \prod_{k=2}^{r-1} \mathbf{D}_{k} \prod_{l=1}^{r-k} \mathbf{G}_{r-l}^{k, l}\right]-\left[\prod_{l=1}^{r-2} \hat{\mathbf{G}}_{r-l}^{1, l} \prod_{k=2}^{r-1} \hat{\mathbf{D}}_{k} \prod_{l=1}^{r-k} \hat{\mathbf{G}}_{r-l}^{k, l}\right]\right\|_{F}^{2}+\left\|\mathbf{E}_{1}\right\|_{F}^{2}+\left\|\mathbf{E}_{1, r-1}^{\prime} \mathbf{G}_{1}^{1, r-1} \hat{\mathbf{G}}_{1}^{1, r-1}\right\|_{F}^{2} \\
& =\left\|\left[\prod_{l=1}^{r-2} \mathbf{G}_{r-l}^{1, l} \prod_{k=2}^{r-1} \mathbf{D}_{k} \prod_{l=1}^{r-k} \mathbf{G}_{r-l}^{k, l}\right]-\left[\prod_{l=1}^{r-2} \hat{\mathbf{G}}_{r-l}^{1, l} \prod_{k=2}^{r-1} \hat{\mathbf{D}}_{k} \prod_{l=1}^{r-k} \hat{\mathbf{G}}_{r-l}^{k, l}\right]\right\|_{F}^{2}+\left\|\mathbf{E}_{1}\right\|_{F}^{2}+\left\|\mathbf{E}_{1, r-1}^{\prime}\right\|_{F}^{2}
\end{aligned}
$$

We now observe that successive application of the triangle inequality yields norms of matrices of the form $\mathbf{E}_{i}$ and $\mathbf{E}_{k, l}^{\prime}$. These are easy to characterize. $\mathbf{E}_{i}$ is a diagonal matrix whose diagonal entries consist of $r-k 1 \mathrm{~s}$ followed by terms $e^{\phi_{k, k}}-e^{\hat{\phi}_{k, k}}, e^{\phi_{k,+1}}-e^{\hat{\phi}_{k, k+1}} \ldots e^{\phi_{k, r}}-e^{\hat{\phi}_{k, r}}$. The $\mathbf{E}_{k, l}^{\prime}$ matrix has all zeros except for four entries, where two of them are $\cos \theta_{k, l}-\cos \hat{\theta}_{k, l}$, and the other two $\sin \theta_{k, l}-\sin \hat{\theta}_{k, l}$. Using this, on further simplification, we get

$$
\operatorname{Tr}(\mathbf{Q}) \leq \sum_{i=1}^{r-1}\left\|\mathbf{E}_{i}\right\|_{F}^{2}+\sum_{k=1}^{r-1} \sum_{l=1}^{r-k}\left\|\mathbf{E}_{k, l}^{\prime}\right\|_{F}^{2}
$$

where $\mathbf{E}_{i}=\mathbf{D}_{i}-\hat{\mathbf{D}}_{i}$ and $\mathbf{E}_{k, l}^{\prime}=\mathbf{G}_{r-l}^{k, l}-\hat{\mathbf{G}}_{r-l}^{k, l}$. We now note that each of

$$
\begin{aligned}
\mathbb{E}\left[\left\|\mathbf{E}_{i}\right\|_{F}^{2}\right] & =\mathbb{E}\left[\operatorname{Tr}\left(\mathbf{D}_{i}-\hat{\mathbf{D}}_{i}\right)\left(\mathbf{D}_{i}-\hat{\mathbf{D}}_{i}\right)^{\dagger}\right] \\
& =\mathbb{E}\left[\sum_{l=i}^{r}\left\|e^{j \phi_{i, l}}-e^{j \hat{\phi}_{i, l}}\right\|^{2}\right] \\
& =\sum_{l=i}^{r} \mathbb{E}\left[\left\|e^{j \phi_{i, l}}-e^{j \hat{\phi}_{i, l}}\right\|^{2}\right] .
\end{aligned}
$$

Under uniform quantization of $\phi_{i, j}$

$$
\mathbb{E}\left[\left\|e^{j \phi_{i, l}}-e^{j \hat{\phi}_{i, l}}\right\|^{2}\right]=2-2 \operatorname{sinc}\left(2^{-b_{1}}\right), \forall l=1 \ldots r, i=l \ldots r-1 .
$$

Thus, we get

$$
\mathbb{E}\left[\left\|\mathbf{E}_{i}\right\|_{F}^{2}\right]=2 r\left(1-\operatorname{sinc}\left(2^{-b_{1}}\right)\right)
$$


Now $\mathbb{E}\left[\left\|\mathbf{E}_{k, l}^{\prime}\right\|_{F}^{2}\right]=\mathbb{E}\left[\left\|\mathbf{G}_{i}^{k, l}-\hat{\mathbf{G}}_{i}^{k, l}\right\|_{F}^{2}\right]$ can be evaluated as

$$
\begin{aligned}
\mathbb{E}\left[\left\|\mathbf{E}_{k, l}^{\prime}\right\|_{F}^{2}\right] & =2 \mathbb{E}\left[\left\|\cos \theta_{k, l}-\cos \hat{\theta}_{k, l}\right\|^{2}+\left\|\sin \theta_{k, l}-\sin \hat{\theta}_{k, l}\right\|^{2}\right] \\
& =4 \mathbb{E}\left[1-\cos \left(\theta_{k, l}-\hat{\theta}_{k, l}\right)\right] \\
& =8 \mathbb{E}\left[\sin \left(\frac{\theta_{k, l}-\hat{\theta}_{k, l}}{2}\right)^{2}\right]
\end{aligned}
$$

Then, from (20), 21) and (22), we get

$$
\mathbb{E}[\operatorname{Tr}(\mathbf{Q})]=\epsilon_{\text {Lloyd }}^{2} \leq r(r-1)\left(1-\operatorname{sinc}\left(2^{-b_{1}}\right)\right)+\sum_{k=1}^{r-1} \sum_{l=1}^{r-k} 8\left(\mathbb{E}\left[\sin \left(\frac{\theta_{k, l}-\hat{\theta}_{k, l}}{2}\right)^{2}\right]\right) .
$$

Since

$$
\frac{\theta_{k, l}-\hat{\theta}_{k, l}}{2} \geq \sin \left(\frac{\theta_{k, l}-\hat{\theta}_{k, l}}{2}\right),
$$

the MSE $\epsilon_{\theta_{l}}^{2}$ for $\theta_{k, l}$ can be bounded as

$$
\epsilon_{\theta_{l}}^{2}=\mathbb{E}\left[\left(\theta_{k, l}-\hat{\theta}_{k, l}\right)^{2}\right] \geq 4 \mathbb{E}\left[\sin \left(\frac{\theta_{k, l}-\hat{\theta}_{k, l}}{2}\right)\right]^{2} .
$$

Recall that the density function of $\theta_{k, l}$ is independent of $k$, from Equation (7). Combining (23) and (24) completes the proof of the theorem.

\section{Appendix B}

\section{Expected Post-coder Quantization Error for $r=2$}

In the following proof, we consider the decomposition for the precoder to be similar to the one in [13], which involves the form that we have used in Section III-A.

Denoting the post-coder quantization error $\mathbf{Q}=\left(\mathbf{U}^{\dagger} \hat{\mathbf{U}}-\mathbf{I}_{r}\right)^{\dagger}\left(\mathbf{U}^{\dagger} \hat{\mathbf{U}}-\mathbf{I}_{r}\right)$ by the matrix $\mathbf{Q}$,

$$
\mathbb{E}[\mathbf{Q}]=2 \mathbf{I}_{r}-\mathbb{E}\left[\mathbf{U}^{\dagger} \hat{\mathbf{U}}\right]-\mathbb{E}\left[\hat{\mathbf{U}}^{\dagger} \mathbf{U}\right]
$$

To evaluate the above, we use the fact that all $2 \times 2$ unitary matrices can be represented using Givens rotations and Householder transformations as follows (with the final diagonal unitary matrix not represented due to redundancy, as discussed shown in Equation (6)):

$$
\mathbf{U}=\underbrace{\left[\begin{array}{cc}
1 & 0 \\
0 & e^{j \phi_{1}}
\end{array}\right]}_{\mathbf{U}_{1}} \underbrace{\left[\begin{array}{cc}
\cos \theta & \sin \theta \\
-\sin \theta & \cos \theta
\end{array}\right]}_{\mathbf{U}_{2}}
$$


Note that we refer to the component matrices as $\mathbf{U}_{1}$ and $\mathbf{U}_{2}$ as marked in the equation (26) for convenience. Therefore, $\phi_{1}$, and $\theta$ uniquely parameterize all $2 \times 2$ unitary post-coders. Since we use scalar quantization to quantize these parameters, the matrix $\hat{\mathbf{U}}$ obtained by reconstruction using the corresponding quantized $\hat{\phi}_{1}$ and $\hat{\theta}$ is

$$
\hat{\mathbf{U}}=\underbrace{\left[\begin{array}{cc}
1 & 0 \\
0 & e^{j \hat{\phi}_{1}}
\end{array}\right]}_{\hat{\mathbf{U}}_{1}} \underbrace{\left[\begin{array}{cc}
\cos \hat{\theta} & \sin \hat{\theta} \\
-\sin \hat{\theta} & \cos \hat{\theta}
\end{array}\right]}_{\hat{\mathbf{U}}_{2}} .
$$

We can use the component matrices $\mathbf{U}_{1}$ and $\mathbf{U}_{2}$ in Equation 26 and $\hat{\mathbf{U}}_{1}$ and $\hat{\mathbf{U}}_{2}$ in Equation (27) to evaluate the expectation in Equation (25). This allows us to exploit the independence of the parameters in computing the expectation. We first consider the matrix $\mathbf{U}^{\dagger} \hat{\mathbf{U}}=\mathbf{U}_{2}^{\dagger} \mathbf{U}_{1}^{\dagger} \hat{\mathbf{U}}_{1} \hat{\mathbf{U}}_{2}$. Since $\hat{\mathbf{U}}_{1}^{\dagger} \mathbf{U}_{1}$ depends only on $\phi_{1}$, this expectation can be evaluated separately as

$$
\mathbb{E}\left[\mathbf{U}_{1}^{\dagger} \hat{\mathbf{U}}_{1}\right]=\left[\begin{array}{cc}
1 & 0 \\
0 & \mathbb{E}\left[e^{j\left(\hat{\phi}_{1}-\phi_{1}\right)}\right]
\end{array}\right]
$$

If we use a uniform $b$-bit quantizer for each $\phi_{1}$ that has $2^{b}$ equi-spaced levels in $(-\pi, \pi]$, then we obtain $\mathbb{E}\left[\mathbf{U}_{1}^{\dagger} \hat{\mathbf{U}}_{1}\right]=\operatorname{diag}\left\{1, \operatorname{sinc}\left(2^{-b}\right)\right\}$. This simplifies the evaluation of the expectation of $\mathbf{Q}$, since, using the rule of iterated expectations and that $\mathbb{E}[\mathbf{Q}]=\mathbb{E}\left[\mathbf{U}_{2}^{\dagger} \mathbb{E}\left[\mathbf{U}_{1}^{\dagger} \hat{\mathbf{U}}_{1} \mid \mathbf{U}_{2}\right] \hat{\mathbf{U}}_{2}\right]$, we get

$$
\mathbb{E}[\mathbf{Q}]=\left[\begin{array}{ll}
\mathbb{E}\left[\cos (\theta) \cos (\hat{\theta})+\sin (\theta) \sin (\hat{\theta}) \operatorname{sinc}\left(2^{-b}\right)\right] & \mathbb{E}\left[\cos (\hat{\theta}) \sin (\theta)-\cos (\theta) \sin (\hat{\theta}) \operatorname{sinc}\left(2^{-b}\right)\right] \\
\mathbb{E}\left[\cos (\theta) \sin (\hat{\theta})-\cos (\hat{\theta}) \sin (\theta) \operatorname{sinc}\left(2^{-b}\right)\right] & \mathbb{E}\left[\sin (\theta) \sin (\hat{\theta})+\cos (\theta) \cos (\hat{\theta}) \operatorname{sinc}\left(2^{-b}\right)\right]
\end{array}\right]
$$

Therefore, substituting this into Equation 25], and taking trace, we get

$$
\epsilon_{\text {Lloyd }}^{2}=\mathbb{E}[\operatorname{Tr}(\mathbf{Q})]=4-2 \mathbb{E}[\cos (\theta-\hat{\theta})]\left(1+\operatorname{sinc}\left(2^{-b}\right)\right)
$$

which is the desired result.

\section{Appendix C}

LOWER BOUND ON ACHIEVAble Rates

Expressing $\mathbf{H}=\mathbf{U} \Sigma \mathbf{V}^{\dagger}$ by SVD, and taking $\tilde{\mathbf{x}}=\mathbf{V x}$, we get

$$
\tilde{\mathbf{y}}=\mathbf{U} \Sigma \mathbf{V}^{\dagger} \tilde{\mathbf{x}}+\tilde{\eta}=\mathbf{U} \Sigma \mathbf{x}+\eta
$$

Let $y=\hat{\mathbf{U}}^{\dagger} \tilde{\mathbf{y}}$, where $\hat{\mathbf{U}}$ is the quantized version of $\mathbf{U}$ available at the UE. We have

$$
\mathbf{y}=\hat{\mathbf{U}}^{\dagger} \mathbf{U} \Sigma \mathbf{x}+\hat{\mathbf{U}}^{\dagger} \tilde{\eta}=\hat{\mathbf{U}}^{\dagger} \mathbf{U} \Sigma \mathbf{x}+\mathbf{w}
$$


where $\mathbf{w}=\hat{\mathbf{U}}^{\dagger} \eta$. Notice that $\mathbf{w}$ and $\eta$ are identically distributed, and independent of $(\mathbf{x}, \mathbf{H})$. We have assumed $\eta \sim \mathcal{N} C(0, \mathbf{I})$. Now, instead of the optimization in (3), one can equivalently consider maximizing the mutual information $I(\mathbf{x} ; \mathbf{y} \mid \Sigma, \hat{\mathbf{U}})$, under the constraint $\mathbb{E}\left[\mathbf{x}^{\dagger} \mathbf{x}\right] \leq P_{T}$. As mentioned earlier, the exact nature of the optimal distribution is unclear in the absence of full CSIR, and appears difficult to characterize. Nevertheless, notice that $\mathrm{C}_{\text {full CSI }}$ is an upperbound to the achievable rate here, thus, we can focus on a lowerbound which is close enough to $\mathrm{C}_{\text {full CSI }}$ itself. In particular, the choice $\mathbf{x} \sim \mathcal{C N}\left(0, \mathbf{K}_{\Sigma}\right)$, for an appropriate covariance matrix $\mathbf{K}_{\Sigma}$, will be shown to achieve rates close to the capacity as the quantization gets finer. The achievable rate $R$ then is given by

$$
\begin{aligned}
R=I(\mathbf{x} ; \mathbf{y} \mid \Sigma, \hat{\mathbf{U}}) & =h(\mathbf{y} \mid \Sigma, \hat{\mathbf{U}})-h(\mathbf{y} \mid \Sigma, \hat{\mathbf{U}}, \mathbf{x}) \\
& \geq h(\mathbf{y} \mid \Sigma, \hat{\mathbf{U}}, \mathbf{U})-h(\mathbf{y} \mid \Sigma, \hat{\mathbf{U}}, \mathbf{x}) \\
& =h(\mathbf{U} \Sigma \mathbf{x}+\eta \mid \Sigma, \mathbf{U}, \hat{\mathbf{U}})-h(\mathbf{y} \mid \Sigma, \hat{\mathbf{U}}, \mathbf{x})
\end{aligned}
$$

The inequality follows from the fact that conditioning reduces differential entropy. Notice that $(\Sigma, \mathbf{U}, \hat{\mathbf{U}}) \rightarrow \mathbf{U} \Sigma \rightarrow \mathbf{U} \Sigma x+\eta$ forms a Markov chain in this order. Thus,

$$
\begin{aligned}
R & \geq h(\mathbf{U} \Sigma \mathbf{x}+\eta \mid \mathbf{U} \Sigma)-h(\mathbf{y} \mid \Sigma, \hat{\mathbf{U}}, \mathbf{x}) \\
& =h(\mathbf{U} \Sigma \mathbf{x}+\eta \mid \mathbf{U} \Sigma)-r \log (\pi e)-[h(\mathbf{y} \mid \Sigma, \hat{\mathbf{U}}, \mathbf{x})-r \log (\pi e)] \\
& =\mathrm{R}_{\text {full-CSI }}-h(\mathbf{y} \mid \Sigma, \hat{\mathbf{U}}, \mathbf{x})+r \log (\pi e),
\end{aligned}
$$

where $\mathbf{R}_{\text {full-CSI }}$ is the achievable rate when $\mathbf{H}$ is known fully at the transmitter and receiver, while taking $\mathbf{x} \sim \mathcal{C N}\left(0, \mathbf{K}_{\Sigma}\right)$. Let us now find a suitable upperbound to the term $h(\mathbf{y} \mid \Sigma, \hat{\mathbf{U}}, \mathbf{x})$. Rewriting (29) we get,

$$
\begin{aligned}
h(\mathbf{y} \mid \Sigma, \hat{\mathbf{U}}, \mathbf{x}) & =h\left(\Sigma \mathbf{x}+\left(\hat{\mathbf{U}}^{\dagger} \mathbf{U}-\mathbf{I}_{r}\right) \Sigma \mathbf{x}+\mathbf{w} \mid \Sigma, \hat{\mathbf{U}}, \mathbf{x}\right) \\
& =h\left(\left(\hat{\mathbf{U}}^{\dagger} \mathbf{U}-\mathbf{I}_{r}\right) \Sigma \mathbf{x}+\mathbf{w} \mid \Sigma, \hat{\mathbf{U}}, \mathbf{x}\right) \\
& \left.\leq h\left(\hat{\mathbf{U}}^{\dagger} \mathbf{U}-\mathbf{I}_{r}\right) \Sigma \mathbf{x}+\mathbf{w}\right) .
\end{aligned}
$$

Applying the entropy maximizing property of Gaussian distribution under a covariance constraint

$$
h(\mathbf{y} \mid \Sigma, \hat{\mathbf{U}}, \mathbf{x}) \leq \mathbb{E}\left[\log \left|\pi e\left(\left(\hat{\mathbf{U}}^{\dagger} \mathbf{U}-\mathbf{I}_{r}\right) \Sigma K_{\Sigma} \Sigma^{\dagger}\left(\hat{\mathbf{U}}^{\dagger} \mathbf{U}-\mathbf{I}_{r}\right)^{\dagger}+N_{o} \mathbf{I}_{r}\right)\right|\right] .
$$

Let us denote $B=\hat{\mathbf{U}}^{\dagger} \mathbf{U}-\mathbf{I}_{r}$. Since $\operatorname{det}(\mathbf{I}+\mathbf{A D})=\operatorname{det}(\mathbf{I}+\mathbf{D A})$ when both AD and DA exist,

$$
h(\mathbf{y} \mid \Sigma, \hat{\mathbf{U}}, \mathbf{x}) \leq \mathbb{E}\left[\log _{2}\left\{\operatorname{det}\left(\pi e\left(\mathbf{I}_{r}+\Sigma \mathbf{K}_{\Sigma} \Sigma^{\dagger} \mathbf{B}^{\dagger} \mathbf{B}\right)\right)\right\}\right]
$$


Notice that $\mathbf{B}^{\dagger} \mathbf{B}=\mathbf{Q}$ is a matrix that captures the error between $\mathbf{U}$ and $\hat{\mathbf{U}}$. Denoting $\mathbf{J}=\Sigma \mathbf{K}_{\Sigma} \Sigma^{\dagger}$,

$$
\begin{aligned}
h(\mathbf{y} \mid \Sigma, \hat{\mathbf{U}}, \mathbf{x}) & \leq \mathbb{E}\left[\log _{2}\left\{\operatorname{det}\left(\pi e\left(\mathbf{I}_{r}+\mathbf{J Q}\right)\right)\right\}\right] \\
& \leq \mathbb{E} \log _{2}\left\{\prod_{i=1}^{r} \pi e\left(1+\mathbf{J}_{i i} \mathbf{Q}_{i i}\right)\right\},
\end{aligned}
$$

by applying the Hadamard's inequality [20] for positive semidefinite matrices. Now applying Jensen's inequality

$$
h(\mathbf{y} \mid \Sigma, \hat{\mathbf{U}}, \mathbf{x}) \leq \sum_{i=1}^{r}\left[\log _{2}\left(1+\mathbb{E}\left\{\mathbf{J}_{i i}\right\} \mathbb{E}\left\{\mathbf{Q}_{i i}\right\}\right)\right]+r \log (\pi e),
$$

since the logarithm is a concave function. Notice that the expectations over $\mathbf{J}_{i i}$ and $\mathbf{Q}_{i i}$ can be separated since they are independent ( $\mathbf{J}$ depends only on $\Sigma$, while $\mathbf{Q}$ depends only on $\mathbf{U})$. From (33) and 37)

$$
I(\mathbf{x} ; \mathbf{y} \mid \Sigma) \geq \mathrm{R}_{\text {full-CSI }}-\sum_{i i=1}^{r}\left[\log _{2}\left(1+\mathbb{E}\left\{\mathbf{J}_{i i}\right\} \mathbb{E}\left\{\mathbf{Q}_{i i}\right\}\right)\right] .
$$

Let us take the covariance of $\mathbf{x}$ as $\mathbf{K}_{\Sigma}=\operatorname{diag}\left[P_{1}, \cdots, P_{r}\right]$, with

$$
P_{n}=\left(\frac{1}{\lambda}-\frac{1}{\sigma_{n}^{2}}\right)^{+}
$$

where $\lambda$ is determined by $(13)$, and $(a)^{+}:=\max \{0, a\}$. Using $(15)$, we get $\mathbb{E}\left[\mathbf{J}_{i i}\right]=P_{\mathrm{rx}}, 1 \leq i \leq r$. Thus

$$
\sum_{i=1}^{r} \log _{2}\left(1+\mathbb{E}\left\{\mathbf{J}_{i i}\right\} \mathbb{E}\left\{\mathbf{Q}_{i i}\right\}\right)=\sum_{i=1}^{r} \log _{2}\left(1+P_{\mathrm{rx}} \mathbb{E} \mathbf{Q}_{i i}\right) .
$$

Now, Jensen's inequality for logsum implies that

$$
\begin{aligned}
\sum_{i=1}^{r} \log _{2}\left(1+P_{\mathrm{rx}} \mathbb{E}\left\{\mathbf{Q}_{i i}\right\}\right) & \leq r \log _{2}\left(\frac{1}{r} \sum_{i=1}^{r}\left(1+P_{\mathrm{rx}} \mathbb{E}\left\{\mathbf{Q}_{i i}\right)\right\}\right) \\
& =r \log _{2}\left(1+\frac{P_{\mathrm{rx}}}{r} \mathbb{E} \operatorname{Tr}(\mathbf{Q})\right) .
\end{aligned}
$$

Since $\operatorname{Tr}(\mathbf{Q})=\|\mathbf{U}-\hat{\mathbf{U}}\|_{F}^{2}$, and $\mathrm{R}_{\text {full-CSI }}=\mathrm{C}_{\text {full CSI }}$ under (39), the rate expression in (38) yields

$$
I(\mathbf{x} ; \mathbf{y} \mid \Sigma) \geq C_{\text {full CSI }}-r \log _{2}\left(1+\frac{P_{\mathrm{rx}}}{r} \mathbb{E}\|\mathbf{U}-\hat{\mathbf{U}}\|_{F}^{2}\right) .
$$

This completes the proof of Theorem 3 . 